\title{
11 COMMUNICATIONS SATELLITE SERVICES
}

The best communications satellites in the world are useless without users. Improving a satellite's lifetime, or its irradiated power, or its stabilization system are all interesting and desirable developments, but the end user has only a few very basic concerns. Will the satellite work and reliably so? Will the capacity needed to provide the service be available on time? Will the satellite provide high quality service on a consistent basis? To many users, the transmission mode is a matter of indifference. You could use a satellite, or a microwave relay, or a coaxial cable, or a pair of twisted wires; all would be quite acceptable as long as they worked. Even a pair of tin cans connected by a wire would be acceptable if the service came through on time. Fortunately satellites work much better than tin cans, and in fact are the first media capable of providing global ISDN quality digital communications to business users and public switched networks.

Because the perspective of the service user is different, this section is devoted to the user community which defines what it is that communications satellites must acutally do. Included here are the views of the international press community, the video conference user groups and broadcasters.

$11 \mathrm{~A}$ Video and Audio Conferencing Networks

Louis A. Bransford and Suzanne G. Douglas

11B Telecommunications Satellites and the Press Today

Oliver Robinson and Louis A. Bransford

Peter Marshall 